\title{
Example of Structure Modeling and Analysis of Ultrasound Scattering for Trabecular Bone
}

\author{
Janusz WÓJCIK, Jerzy LITNIEWSKI, \\ Andrzej NOWICKI \\ Institute of Fundamental Technological Research \\ Polish Academy of Sciences \\ Pawińskiego 5B, 02-106 Warszawa, Poland \\ e-mail: jwojcik@ippt.gov.pl \\ (received September 16, 2010; accepted November 10, 2010)
}

\begin{abstract}
A trabecular bone consists of trabeculae whose mechanical properties differ significantly from the surrounding marrow, therefore an ultrasonic wave propagating within the bone structure is strongly scattered. The aim of this paper was to evaluate the contribution of the first, second and higher order scattering (multiple scattering) into the total scattering of ultrasound in a trabecular bone. The scattering due to the interconnections between thick trabeculae, usually neglected in trabecular bone models, has been also studied. The basic element in our model of the trabecular bone was an elastic cylinder with a various finite-length and diameter as well as orientation. The applied model was taking into account variation of both, elements size and their spatial configuration. The field scattered on the bone model was evaluated by solving numerically the integral form of the generalized Sturm-Liouville equation describing a scalar wave in inhomogeneous and lossy media. For the scattered fields calculated numerically the effective cross-sections were determined. The influence of absorption on the scattering coefficients was demonstrated. The results allowed to conclude that within the frequency range from 0.5 to $1.5 \mathrm{MHz}$ contribution of the second order scattering to the effective backscattering cross-section is at least 500 times lower than that due to the first order scattering. It was noticed that for a frequency higher than $1.5 \mathrm{MHz}$ fast growth of the backscattering (reflection) coefficients, calculated for the second order scattering, occurs.
\end{abstract}

Keywords: multi-scattering, random complex structures, trabecular bone model.

\section{Introduction}

The evaluation of strength of the bone requires knowledge not only of its mean density but also of its microscopic structure. The ultrasonic signals scattered in 
the trabecular bone contain information about properties of the bone structure, therefore analysis of the backscatter could be useful in assessment of the microscopic architecture of the bone. It has been demonstrated that usage of the backscattering models of the bone enables an assessment of some micro-structural characteristics from the experimental data.

There are several publications considering various scattering models in the trabecular bone (WEAR, 1999; CHAFFAi et al., 2000; LitNiEWSKi et al., 2009; LUGier et al., 2008; LiTNIEWSKI, 2010) but, to the best of authors' knowledge, all of the reported bone scattering models assumed the Born approximation, that neglected the multiple scattering within the bone trabeculae. The trabecular bone consists of trabeculae whose mechanical properties differ significantly from those of the surrounding marrow and therefore the ultrasonic wave is strongly scattered. The work of Bossy et al. (2005) presents the analytically advanced approach. The scattering structure corresponds to the real one. Unfortunately this approach does not enable determination of influence of the multiple scattering on the total field. The Wear's (2008) work contains the review of methods and problems of bone sonometry.

The aim of the presented paper was evaluation of contribution of the first, second and higher order scattering (multiple scattering) into the total scattering of the ultrasonic signals in the trabecular bone. The scattering due to interconnections between thick trabeculae, usually neglected in trabecular bone models, has been also studied. Our model is fully scaled.

The basic element in our model of the trabecular bone was an elastic cylinder with a various finite-length and diameter as well as its orientation. Its density and speed of sound were similar to those of the bone tissue. The cylinder was applied in building of the multi-element structures, similar to the architecture of the trabecular bone, taking into account variation of size of the elements and their spatial configuration. The field scattered on the bone model was evaluated by solving numerically the integral form of the generalised SturmLiouville equation that describes the longitudinal wave in inhomogeneous and lossy media.

The mathematical model was formulated in the space-time frequency domain, contrary to the approaches of other authors. Only such approach can easily incorporate the absorption properties of the media with non-classical viscosity. The models describing the absorption losses formulated in the time-space would include the non-local terms in the time complex integral. Their presence would virtually preclude the possibility of the efficient numerical computing.

The images of the fields scattered on the bone structure in function of the incident wave frequency varying from $0.5 \mathrm{MHz}$ up to $3 \mathrm{MHz}$, and up to the third order of scattering are presented. For the calculated scattered fields the effective cross-sections related to the detected echo-signal level, were determined. The calculations were performed for the different absorption parameters and for the frequency range from 0.5 to $3 \mathrm{MHz}$. 


\section{Basic equations}

The Lame's equation for the longitudinal (volumetric) disturbances in the non-homogeneous, isotropic and stationary medium given in (BREKHOVSKIKH, GoDIN, 1990) can be rewritten in the space-Fourier frequency domain as follows

$$
\begin{gathered}
g \nabla \cdot\left(\frac{\nabla C}{g}\right)+K^{2} C=0, \\
K^{2}=K^{2}(\mathbf{x}, n) \equiv \frac{n^{2}}{c^{2}\left(1-2 i\left(a(\mathbf{x}, n) / g c^{2} n\right)\right)} \\
C=\left(\lambda+2 \mu+2 \frac{a(n)}{i n}\right) \nabla \cdot \mathbf{u}_{F}=g c^{2}\left(1+2 \frac{a(n)}{g c^{2} i n}\right) \nabla \cdot \mathbf{u}_{F},
\end{gathered}
$$

where $C=C(\mathbf{x}, n)=F[P(\mathbf{x}, t)], \mathbf{u}_{F}(\mathbf{x}, n)=F[\mathbf{u}(\mathbf{x}, t)] ; F[\cdot]$ is the Fourier transform, $P=P(\mathbf{x}, t)$ is the normalized stress; $P \equiv \bar{P} / P_{0}$, where $P_{0}$ is the reference pressure, $\mathbf{u}=\mathbf{u}(\mathbf{x}, t)$ is the normalized displacement vector; $\lambda+2 \mu=g c^{2}$, where $\lambda=\lambda(\mathbf{x}), \mu=\mu(\mathbf{x})$ are the first and the second Lame's constants, respectively; $g=g(\mathbf{x}), c=c(\mathbf{x})$ are the normalized density and speed of the longitudinal (sound) waves, respectively, $(\mathbf{x}, t)$ are normalized coordinates in space and time, whereas $\nabla$ is the normalized nabla vector operator, $\Delta \equiv \nabla \cdot \nabla$ is the scalar Laplacian, where the symbol "." denotes scalar product, $i$ is the imaginary unit. The normalization was performed as follows: $g \equiv \bar{g} / g_{0}, c \equiv \bar{c} / c_{0}, \mathbf{u} \equiv K_{0} \overline{\mathbf{u}}, \mathbf{x} \equiv K_{0} \overline{\mathbf{x}}$, $t \equiv \omega_{0} \bar{t}, \nabla \equiv \bar{\nabla} / K_{0}$. The dimensional variables and operators are accented; $g_{0}$, $c_{0}$ are the density and speed of sound, respectively for the reference medium (in our case - the reference medium is dominant in the volume), $\lambda_{0}+2 \mu_{0}=g_{0} c_{0}^{2}$. It means that $c=1$ and $g=1$ for the reference medium. The characteristic wave number $K_{0}$ and pulsation $\omega_{0}$ are restricted by the relation: $K_{0} c_{0}=\omega_{0}$. $\omega_{0} \equiv 2 \pi / T_{0}$, where $T_{0}$ is the reference time (e.g. time window). A consequence of the applied method of normalization is equality of the non-dimensional pulsation $n \equiv \omega / \omega_{0}$ and frequency, as well as the wave number in the non-dispersive media $k(n)= \pm n$. In homogeneous regions of the medium $a(n)=a(\mathbf{x}, n)$; here $a(\mathbf{x}, n)$ denotes spatial distribution of the small-signal absorption coefficient.

The investigation of influence of the absorption on the wave propagation in numerous media (especially in biological) require generalization of the constitutive relations $C=\left(\lambda+2 \mu-2 i \alpha_{2} n\right) \nabla \cdot \mathbf{u}_{F} ; P=\left(\lambda+2 \mu+2 \alpha_{2} \partial_{t}\right) \nabla \cdot \mathbf{u}$; where $\alpha_{2} \equiv\left(\eta_{b}+4 \eta_{s h} / 3\right) / 2$. Here $\eta_{s h} \equiv \bar{\eta}_{s h} \omega_{0} / g_{0} c_{0}^{2}, \eta_{b} \equiv \bar{\eta}_{b} \omega_{0} / g_{0} c_{0}^{2}$ are the normalized share and bulk viscosities, respectively, describing classically (viscous) absorbing media. We propose this generalization in the form given by Eq. (2) (WóJCIK, 1998). For $a(n)=\alpha_{2} n^{2}$ we obtain classically absorbing (viscous) media (KAUfMAN et al., 2008).

The simplification of the full Lame's equation to the form presented by Eq. (1) depends on the analytical properties of the assumed heterogeneity model (because in full Lame's equation the term $\mathbf{u} \Delta \mu$ occurs, then the only assumption $\nabla \times \mathbf{u}=\mathbf{0}$ 
is not sufficient for derivation of Eq. (1)). The next section will present the model of the heterogeneous medium with material parameters changing by step rise. For this model $\Delta \mu=0$ also on the surfaces of the phase separation, despite the fact that $\nabla \mu \neq 0$ on these surfaces. We rewrite Eq. (1) in the form of the SturmLiouville equation,

$$
\begin{gathered}
\Delta C+k^{2} C=\mathrm{V} C+\mathbf{Q} \cdot \nabla C, \\
k^{2}=k^{2}(n) \equiv n^{2} /\left(1-2 i\left(a_{0}(n) / n\right)\right), \\
\mathrm{V}=\mathrm{V}(\mathbf{x}, n) \equiv-\left(K^{2}(x, n)-k^{2}(n)\right), \quad \mathbf{Q}(\mathbf{x}) \equiv \nabla g / g,
\end{gathered}
$$

where $a_{0}(n)$ is the absorption coefficient of the reference medium.

\section{Medium model description}

We assume that the reference homogeneous medium surrounds $L$ regions $\left(v_{l^{-}}\right.$ regions) in space. The regions are bounded by the surfaces $s_{l}, l=1, \ldots, L$. We suppose that the $v_{l}$ are the open sets in space, however the $\bar{v}_{l}=v_{l} \cup s_{l}$ are closed. Each region $\bar{v}_{l}$ is filled with other homogeneous medium with normalized density, $g_{l} \neq 1$, and speed of the longitudinal wave $c_{l} \neq 1$. The multiple-theory sum of the $v_{l}$ sets describes the structure being submerged in the reference medium. We assume that the elements of structure do not cross in a sense of 3D measure of volume $d^{3}(\cdot)$ however they may be tangential $\left(v=\bigcup_{l} v_{l}, s=\bigcup_{l} s_{l}, d^{3}\left(v_{l} \cap v_{m}\right)=0\right.$, $\left.d^{2,1}\left(\bar{v}_{l} \cap \bar{v}_{m}\right) \neq 0\right)$. Thus the spatial distributions of the sound speed, density and absorption coefficient have a form:

$$
\begin{aligned}
c(\mathbf{x})^{2} & =1+\sum_{l} \mathrm{~d} c_{l}(\mathbf{x})^{2}=1+\sum_{l} \chi_{l}\left(c_{l}^{2}-1\right), \\
g(\mathbf{x}) & =1+\sum_{l} \mathrm{~d} g_{l}(\mathbf{x})=1+\sum_{l} \chi_{l}\left(g_{l}-1\right), \\
a(\mathbf{x}, n) & =a_{0}(n)+\sum_{l} \mathrm{da}_{l}(\mathbf{x}, n)=a_{0}(n)+\sum_{l} \chi_{l}\left(a_{l}(n)-a_{0}(n)\right),
\end{aligned}
$$

where $\mathrm{d}$ denotes the step rise of the material parameters; $\chi_{l} \equiv \chi\left(\bar{v}_{l}\right)$ is the characteristic function of $\bar{v}_{l}\left(\chi=1\right.$ for $\mathbf{x} \in v_{l}, \chi=0$ for $\mathbf{x} \notin \bar{v}_{l}, \chi=1 / 2$ for $\left.\mathbf{x} \in s_{l}\right)$.

The characteristic function $\chi_{l}(\mathbf{x})$ can be presented in an analytical form as a double layer potential (JACKSON, 1975)

$$
\chi_{l}(\mathbf{x})=-\frac{1}{4 \pi} \int_{s_{l}} \frac{\mathbf{e}_{\mathbf{r}} \cdot \mathbf{e}_{l}}{r\left(\mathbf{x}, \mathbf{x}^{\prime}\right)^{2}} d s_{l}=\frac{1}{4 \pi} \int_{s_{l}} d \theta=\left\{\begin{array}{ll}
1 & \mathbf{x} \in v_{l}, \\
1 / 2 & \mathbf{x} \in s_{l}, \\
0 & \mathbf{x} \notin \bar{v}_{l},
\end{array} \quad \mathbf{x}^{\prime} \in s_{l},\right.
$$


where $r=r\left(\mathbf{x}, \mathbf{x}^{\prime}\right) \equiv|\mathbf{r}|$ (here $\mathbf{r}=\mathbf{x}-\mathbf{x}^{\prime}, \mathbf{e}_{\mathbf{r}}=\nabla r ; \mathrm{d} \theta=-\mathbf{e}_{\mathbf{r}} \cdot \mathbf{e}_{l} \mathrm{~d} s_{l} / r^{2}$ ) is the solid angle under which the surface element $\mathrm{d} s_{l}$ is observed from point $\mathbf{x}$, see Fig. 1.

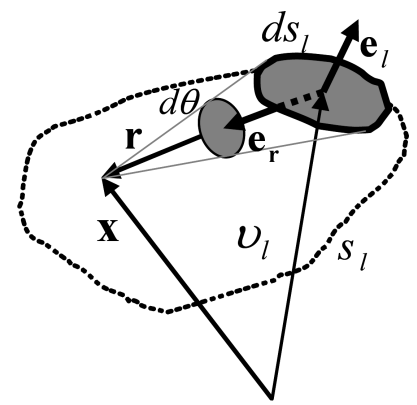

Fig. 1. Relations between vectors for calculations of the Eq. (5).

Since $\Delta \chi_{l}(\mathbf{x})=0$ for all $\mathbf{x}$, then $\Delta g(\mathbf{x})=0$ and $\Delta \mu(\mathbf{x})=0$, despite the fact that $\nabla g \neq 0$ on the surfaces $s$. Omitting the detailed discussion, we have

$$
\begin{array}{r}
\mathrm{V}=\sum_{l} \chi_{l} n^{2}\left(\frac{1}{1-2 i\left(a_{0}(n) / n\right)}-\frac{1}{c_{l}^{2}\left(1-2 i\left(a_{l}(n) / g_{l} c_{l}^{2} n\right)\right)}\right), \\
\mathbf{Q}(\mathbf{x})=\sum_{l}\left(\frac{1-g_{l}}{\left(g_{l}+1\right) / 2}\right) \mathbf{e}_{l}(\mathbf{x}) \delta\left(s_{l}\right) \\
=\mathrm{Q}(\mathbf{x}) \mathbf{e}(\mathbf{x}) \delta(\mathrm{s})=\left(\sum_{l} \sigma_{l}\left(\frac{1-g_{l}}{\left(g_{l}+1\right) / 2}\right)\right) \mathbf{e}(\mathbf{x}) \delta(s) .
\end{array}
$$

Here $\delta(\ldots)$ is the Dirac distribution, $\sigma_{l}$ denotes the characteristic function of the surface $s_{l}$. The vector $\mathbf{e}_{l}(\mathbf{x})$ is externally normal to $s_{l}$ for $\mathbf{x} \in s_{l}$ and is unit. Since $\mathbf{e}(\mathbf{x})=\left\{\mathbf{e}_{l}(\mathbf{x}): \mathbf{e}_{l} \perp s_{l}, l=1, \ldots, L\right\}$ is a general field of the unit vectors being normal to the structure, then: $\delta\left(s_{l}\right) \mathbf{e}(\mathbf{x})=\delta\left(s_{l}\right) \mathbf{e}_{l}(\mathbf{x})$ since for $\mathbf{x} \notin s_{l}, \delta\left(s_{l}\right)=0$.

\section{Scattering equations}

For the assumed model of the medium structure the Eq. (3) becomes as follows

$$
\Delta C+k^{2} C=\mathrm{V} C+\mathrm{Q} B \delta(s),
$$

where $B(\mathbf{x}, n) \equiv \mathbf{e}(\mathbf{x}) \cdot \nabla C(\mathbf{x}, n)$. The field $B$ is determined only on the surface $s$ of the structure $\bar{v}$. Further, if it will not make misunderstanding, the pulsation $n$ will be neglected in the argument list. Transforming the Eq. (8) into the integral equation and using the features of the distributions $\delta(s)$ and $\chi(v)$ we obtain

$$
C(\mathbf{x})=C^{0}(\mathbf{x})-\int_{v} G\left(r\left(\mathbf{x}, \mathbf{x}^{\prime}\right)\right) \mathrm{V}\left(\mathbf{x}^{\prime}\right) C\left(\mathbf{x}^{\prime}\right) d v-\int_{s} G\left(r\left(\mathbf{x}, \mathbf{x}^{\prime}\right)\right) \mathrm{Q}\left(\mathbf{x}^{\prime}\right) B\left(\mathbf{x}^{\prime}\right) d s .
$$


Here $G(r, n) \equiv \exp (i k(n) r / 4 \pi r)$, where $r=r\left(\mathbf{x}, \mathbf{x}^{\prime}\right)=\left|\mathbf{x}-\mathbf{x}^{\prime}\right|, C^{0}(\mathbf{x}) \equiv C^{0}(\mathbf{x}, n)$, is the solution of the Helmholtz equation in the reference medium (incident field), $G\left(r\left(\mathbf{x}, \mathbf{x}^{\prime}\right)\right)=G\left(r\left(\mathbf{x}, \mathbf{x}^{\prime}\right), n\right)$ is the Green function of the Helmholtz equation. Applying $\mathbf{e}(\mathbf{x}) \cdot \nabla$ to the both sides of Eq. (9) the equation for the field $B$ (acceleration) is obtained,

$B(\mathbf{x})=B^{0}(\mathbf{x})-\int_{v} \partial G\left(r\left(\mathbf{x}, \mathbf{x}^{\prime}\right)\right) \mathrm{V}\left(\mathbf{x}^{\prime}\right) C\left(\mathbf{x}^{\prime}\right) d v-\int_{s} \partial G\left(r\left(\mathbf{x}, \mathbf{x}^{\prime}\right)\right) \mathrm{Q}\left(\mathbf{x}^{\prime}\right) B\left(\mathbf{x}^{\prime}\right) d s$,

where $B^{0}=\mathbf{e} \cdot \nabla C^{0}, \partial G(r) \equiv \mathbf{e}(\mathbf{x}) \cdot \mathbf{e}(\mathbf{r}) \partial_{r} G, \mathbf{e}(\mathbf{r})=\nabla r=\mathbf{r} / r$.

The integrals in the Eqs. (9) and (10) describe the scattering of the incident field on the potentials $\mathrm{V}$ and $\mathrm{Q}$ of the structure. It is sufficient to determine the equations for $\mathbf{x} \in \bar{v}$ in order to solve them. When this solution is substituted to the integrals in Eq. (9) the solution for the whole medium is obtained.

Using the grouping of the functions and their normal derivatives in the vector function

$$
\begin{array}{ll}
\overline{\mathrm{C}} \equiv\left(\begin{array}{c}
C \\
B
\end{array}\right), & \overline{\mathrm{C}}^{0} \equiv\left(\begin{array}{c}
C^{0} \\
B^{0}
\end{array}\right), \\
\overline{\mathrm{G}} \equiv\left(\begin{array}{c}
G \\
\partial G
\end{array}\right), & \mathrm{W} \equiv\left(\begin{array}{c}
\mathrm{V} d v \\
\mathrm{Q} d s
\end{array}\right)
\end{array}
$$

and introducing the scattering field $\mathrm{E} \equiv \overline{\mathrm{C}}-\overline{\mathrm{C}}^{0}$ the Eqs. (9) and (10) can be rewritten in the compact form

$$
(\mathbf{I}+\mathbf{G W}) \mathrm{E}=-\mathrm{E}^{0} \quad \mathbf{G} \mathbf{W} \overline{\mathrm{C}} \equiv \int(\overline{\mathrm{G}} \circ \mathrm{W}) \overline{\mathrm{C}},
$$

where the kernel of the operator $\mathbf{G W}$ is the $2 \times 2$ matrice (matrix of matrices) determined by the diadic product of vectors signed by $\circ, \mathrm{E}^{0}=\mathbf{G} \mathbf{W} \overline{\mathrm{C}}^{0}$. $\mathbf{I}$ is the identity operation $\mathbf{I E}\left(\mathbf{x}^{\prime}\right)=\mathrm{E}(\mathbf{x})$. The equivalent form of the operator $\mathbf{G W}$ is

$$
\mathbf{G W} \equiv\left(\begin{array}{ll}
\int d v \mathrm{VG} & \int d s \mathrm{Q} G \\
\int d v \mathrm{~V} \partial G & \int d s \mathrm{Q} \partial G
\end{array}\right) .
$$

The integration domains are clearly determined by both $\mathrm{d} v$ and $\mathrm{d} s$. Since $\mathrm{E}=\sum_{l} \chi_{l} \mathrm{E}=\sum_{l} \mathrm{E}_{l}$, then the operator $\mathbf{G W}$ can be presented as the sum of the cells $\mathbf{G W}=\bigcup_{l, m} \mathbf{G} \mathbf{W}_{l m}$, where $\mathbf{G} \mathbf{W}_{l m}$ is given by Eq. (12) or Eq. (13) for $\mathrm{W}_{m}=\chi_{m} \mathrm{~W}$ and $\mathbf{x} \in \bar{v}_{l}$. For $\mathbf{x}, \mathbf{x}^{\prime} \in \bar{v}_{l}$ and $\mathbf{x}=\mathbf{x}^{\prime}, \mathbf{G W}_{l m}=\mathbf{0}$ (no self interaction). For diagonal cells $l=m$ and we can set $\mathbf{G} \mathbf{W}_{l} \equiv \mathbf{G W}_{l l}$. 


\section{Solution method}

We seek the solution of Eq. (12) for $\mathbf{x} \in v$ in the form

$$
\mathrm{E}=\sum_{l} \mathrm{E}_{l}^{1}+\mathrm{R}^{2}
$$

where $\mathrm{E}_{l}^{1}$ is the solution of Eq. (12) in $l$-th element of the structure assuming that the only scattering field in $\bar{v}_{l}$ is $\mathrm{E}_{l}^{0}=\chi_{l} \mathrm{E}^{0}$ produced by the incident field $\overline{\mathrm{C}}_{l}^{0}=\chi_{l} \overline{\mathrm{C}}^{0}, \mathrm{E}_{l}^{0}=\mathbf{G} \mathbf{W}_{l} \overline{\mathrm{C}}_{l}^{0}$.

$$
\begin{gathered}
\left(\mathbf{I}+\mathbf{G W}_{l}\right) \mathrm{E}_{l}^{1}=-\mathrm{E}_{l}^{0}, \\
\mathrm{E}_{l}^{1}=-\mathbf{H}_{l} \mathrm{E}_{l}^{0}=-\mathbf{H}_{l} \mathbf{G} \mathbf{W}_{l} \bar{C}_{l}^{0}, \quad l=1, \ldots, L,
\end{gathered}
$$

where $\mathbf{H}_{l} \equiv\left(\mathbf{I}+\mathbf{G W}_{l}\right)^{-1}$ denotes the inverse operator. The fields $\mathrm{E}_{l}^{1}$ determine a field in the medium for the first order of scattering (single scattering). The field $\mathrm{E}_{l}^{0}$ represents the first term in the Neumann series obtained by iteration of integral equations (9) and (10). The reminder $\mathrm{R}^{2}$ denotes a field in the structure created due to interaction between the structure elements at the second and higher orders of the scattering (multi-scattering). The $\mathrm{R}^{2}$ satisfies the following equation

$$
(\mathbf{I}+\mathbf{G W}) \mathrm{R}^{2}=-\sum_{l} \mathbf{G W}_{l}\left(\sum_{m, m \neq l} E_{m}^{1}\right), \quad E_{m}^{1}=\mathbf{G W}_{l m} \mathrm{E}_{m}^{1} .
$$

The field $E_{m}^{1}$ is calculated as the field from the $m$-th element failing on the $l$-th element. Then we repeat the procedure described above. We suppose that $\mathrm{R}^{2}=\sum_{l} \mathrm{E}_{l}^{2}+\mathrm{R}^{3}$ and $\mathrm{E}_{l}^{2}$ satisfy Eq. (15) with the source in the form of the $l$-th component on the right hand side in Eq. (17). Then we have

$$
\mathrm{E}_{l}^{2}=-\mathbf{H}_{l} \mathbf{G} \mathbf{W}_{l} \sum_{m \neq l} E_{m}^{1},
$$

and after substitution $E_{m}^{2} \rightarrow E_{m}^{1} \mathrm{R}^{3}$ satisfies Eq. (17).

Generally, at the $j$-th order of the scattering $\mathrm{R}^{j}=\sum_{l} \mathrm{E}_{l}^{j}+\mathrm{R}^{j+1}$,

$$
\mathrm{E}_{l}^{j}=-\mathbf{H}_{l} \mathbf{G} \mathbf{W}_{l} \sum_{m \neq l} E_{m}^{j-1},
$$

then at the point $\mathbf{x}$ of the medium the total $j$-th order component of the scattered field takes the form

$$
E^{j}(\mathbf{x})=-\sum_{l} \mathbf{G W}_{l} \mathrm{E}_{l}^{j}\left(\mathbf{x}^{\prime}\right), \quad \mathbf{x}^{\prime} \in \bar{v}_{l} .
$$

The total scattered field is given by the sum of the $E^{j}(\mathbf{x})$. 
We obtain the discrete (numerical) representation of the above procedure when $\mathrm{W}$ is replaced by the system of the weighted coefficients $\mathrm{W}\left(\zeta_{l}\right)$ for the numerical integration in respect to the vector of structure sampling $\mathbf{x} \rightarrow \mathbf{x}\left(\zeta_{l}\right)$, where $\zeta_{l}$ is the sample index in the $l$-th element of the scattering structure.

\section{Trabecular bone model}

The space skeleton of the model of trabecular bone structure, applied for calculations of the scattering field, is presented in Fig. 2a. One of the skeleton structures, parallel to the $x-z$ plane (horizontal in respect to the incident field), is shown in Fig. 2b. The cylinder with a diameter $\Phi$ and length d was adopted as the model of the trabecular and bar. Each segment of the skeleton is the axis of the cylinder.

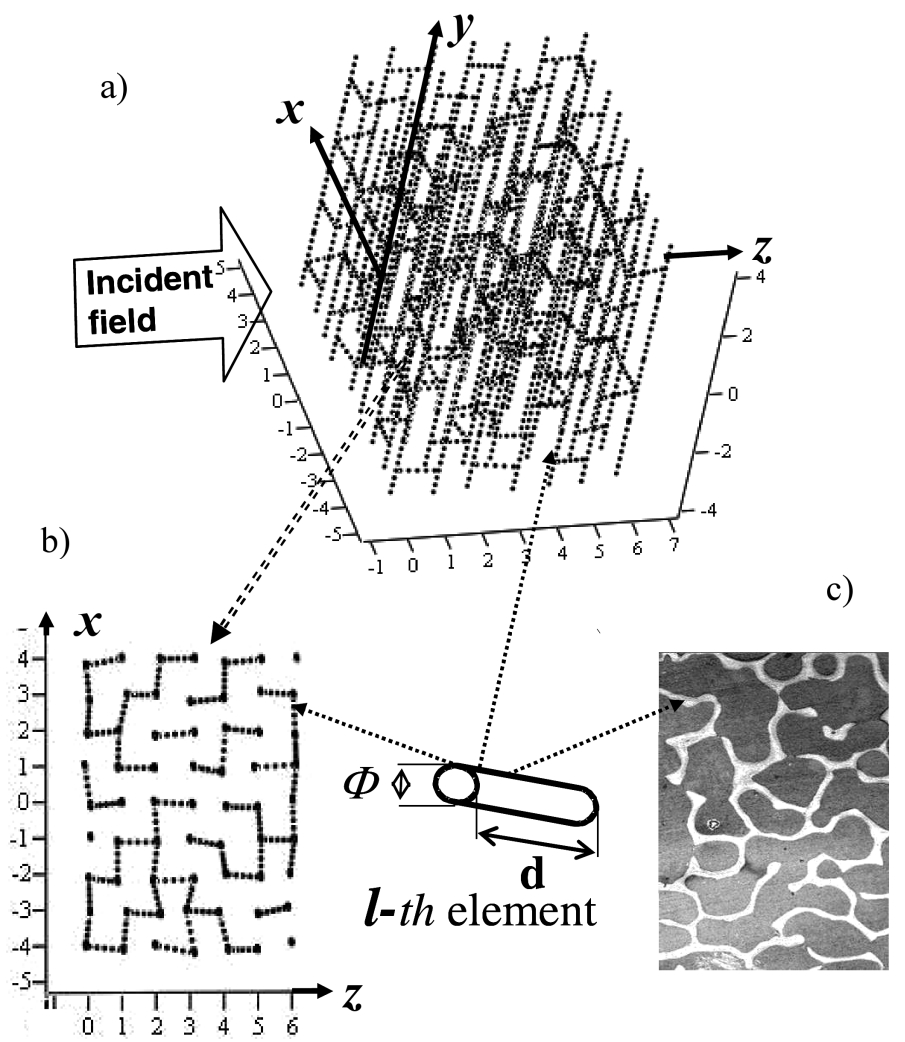

Fig. 2. a) The full skeleton of the trabecular bone model; b) one of the horizontal substructures in the skeleton; c) the cross-section of the real trabecular bone structure.

The skeleton was obtained randomly by displacement of nodes in each layer of a regular structure built of cuboids. The uniform probability was assumed for displacements. The horizontal structures were adjusted to the new node positions 
and next, certain elements were randomly eliminated from the structure. The structure was immersed in the absorbing (or not) fluid filler. The results are very similar to those presented in (HosOKAWA, 2008).

Our model is fully calibratable. The possibility of changing of the number of elements and their geometrical parameters enables modeling the structure with various porosity. Changing the probability distribution functions and their parameters we are able to create the various statistical properties of the structure that account for variation of its physical and geometrical parameters. It should be noted that in our model it is possible to change the shape of the elements, which fill the skeleton (cuboids, spheroids etc.).

\section{Results}

In the initial regular structure the cuboids dimensions are $2 \mathrm{~mm}$ in the $\mathbf{y}$ direction and $1 \mathrm{~mm} \times 1 \mathrm{~mm}$ in the $\mathbf{x}$ and $\mathbf{z}$ directions, respectively. For the displacement of nodes the uniform probability was assumed in the range between -0.15 and $0.15 \mathrm{~mm}$. Some elements were randomly (uniformly) eliminated from the structure.

The normal distribution of the sound speed and density for each trabecula was selected. The maximum deviation from the mean values of $4000 \mathrm{~m} / \mathrm{s}$ and $2000 \mathrm{~kg} / \mathrm{m}^{3}$ was not exceed $\pm 5 \%$. For trabecula in the $\mathbf{y}$ direction and in the horizontal planes the mean values of $\Phi=0.05 \mathrm{~mm}$ and $\Phi=0.04 \mathrm{~mm}$ with the deviations $\pm 20 \%$ and $\pm 25 \%$, respectively, were assumed. For the surrounding medium (marrow - fluid filler) as well as for the surrounding space $g_{0}=1000 \mathrm{~kg} / \mathrm{m}^{3}$, $c_{0}=1500 \mathrm{~m} / \mathrm{s}$ were assumed. The absorption parameter for the fluid filler was equal to $\alpha_{1}=(0.23,1.15$ and 2.3$) \cdot 10^{-4} \mathrm{~Np} /(\mathrm{m} \cdot \mathrm{Hz}), a(n)=\alpha_{1} c_{0}|n| / 2 \pi$. The total number of 433 elements (trabeculae) was considered. The total dimensions were: $[-4 ; 4] \mathrm{mm}$ in the $x$ axis, $[-4 ; 4] \mathrm{mm}$ in the $y$ axis, $[0 ; 6] \mathrm{mm}$ in the $z$ axis $\left(384 \mathrm{~mm}^{3}\right)$.

The unit plane wave was assumed as the incident field $C^{0}=\exp (i k(\nu) z) z \geq 0$, $\nu \in[0.5,3] \mathrm{MHz}$ with the step $0.0333 \mathrm{MHz}$. The dimensionless frequency was $n=15,16, \ldots, 90$.

\subsection{Scattering field distributions}

The exemplary distributions of the scattering fields for the absorption parameter $\alpha_{1}=2.3 \cdot 10^{4} \mathrm{~Np} /(\mathrm{m} \cdot \mathrm{Hz})$ at the subsequent orders for the selected frequencies are shown in Fig. 3. The brightness refers to the logarithmic scale of values. The contour of the scattering structure and its location is shown by means of the red rectangle, whereas the red arrow indicates the direction of the incident wave. The represented area is the rectangle with location $[-30,20] \mathrm{mm}$ in the $z$ axis and $[-15,15] \mathrm{mm}$ in the $x$ axis. 


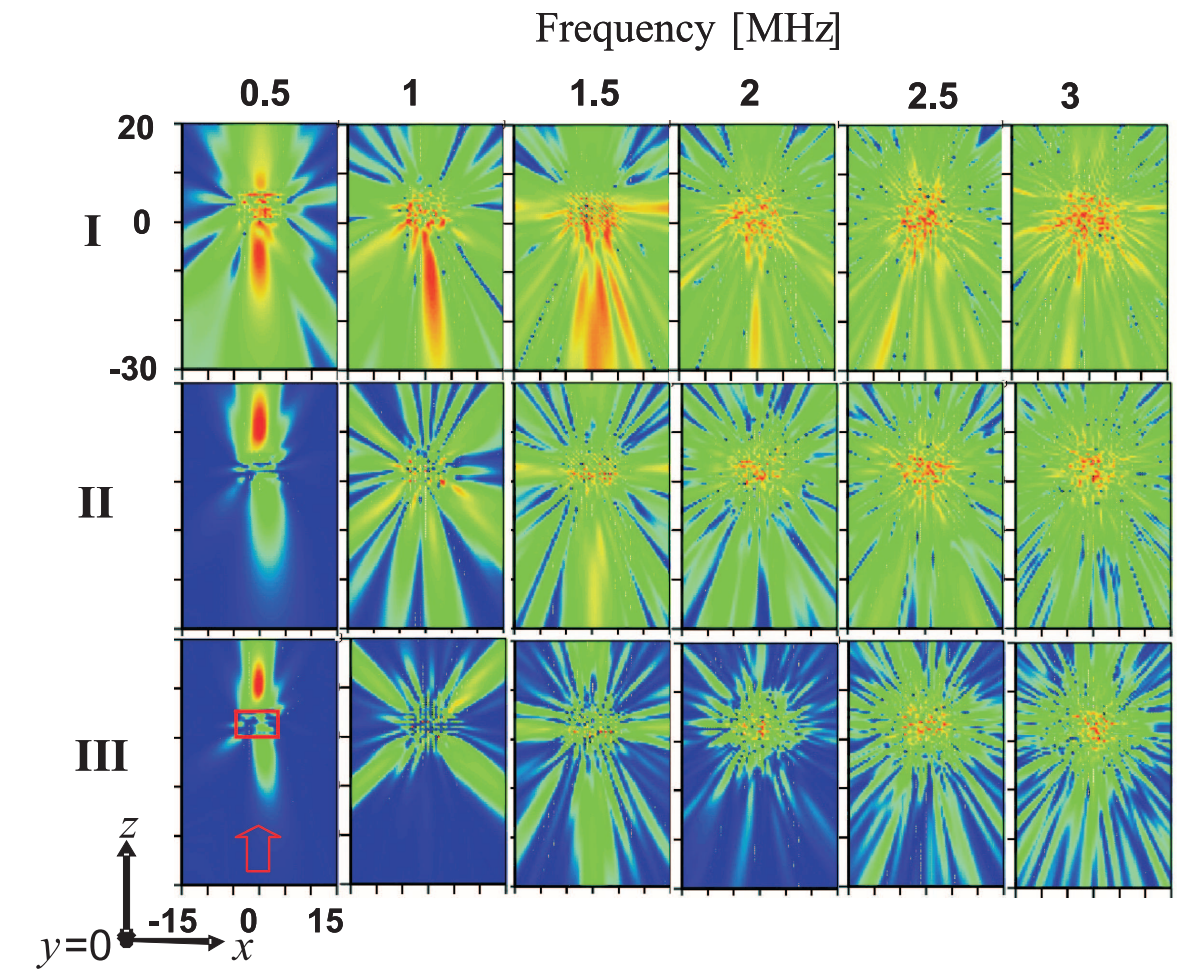

Fig. 3. The distributions of the fields at the subsequent orders of scattering (rows: I, II, III) and as a function of frequency - columns. The axis are in $\mathrm{mm}$.

\subsection{Backscatter coefficients}

We define the horizontal substructure (denoted by "h") as a set of all trabeculae that are situated in the planes being parallel to the $(x, z)$ plane, and the vertical substructure (denoted by ' $\mathbf{y}$ ') as a set of all trabeculae which are parallel to the $y$ axis.

In Fig. 4 the SI, SII and SIII are the backscatter effective cross-section coefficients, that were obtained at the subsequent orders of scattering (first - I, second - II, third - III) and as the function of frequency $\nu$. The influence of the filler absorption on the effective cross-section for each order of scattering is presented. The plots $\boldsymbol{\alpha} 1, \boldsymbol{\alpha} 2, \boldsymbol{\alpha} 3$ correspond to the absorption parameter equal to $\alpha_{1}=0.23 \cdot 10^{-4}, \alpha_{1}=1.15 \cdot 10^{-4}$ and $\alpha_{1}=2.3 \cdot 10^{-4} \mathrm{~Np} /(\mathrm{m} \cdot \mathrm{Hz})$, respectively.

The square roots $\sqrt{\mathbf{S I}}, \sqrt{\mathbf{S I I}}, \sqrt{\mathbf{S I I I}}$ were applied for the better representation. Moreover, they are of the first range in respect to the variations of the scattered field as well as the invariants (as SI, SII, SIII) in respect to the shape of the surface surrounding the scatter in semi-space. Then, the square root from the effective cross-section is a proper measure (norm) of the signal received by 

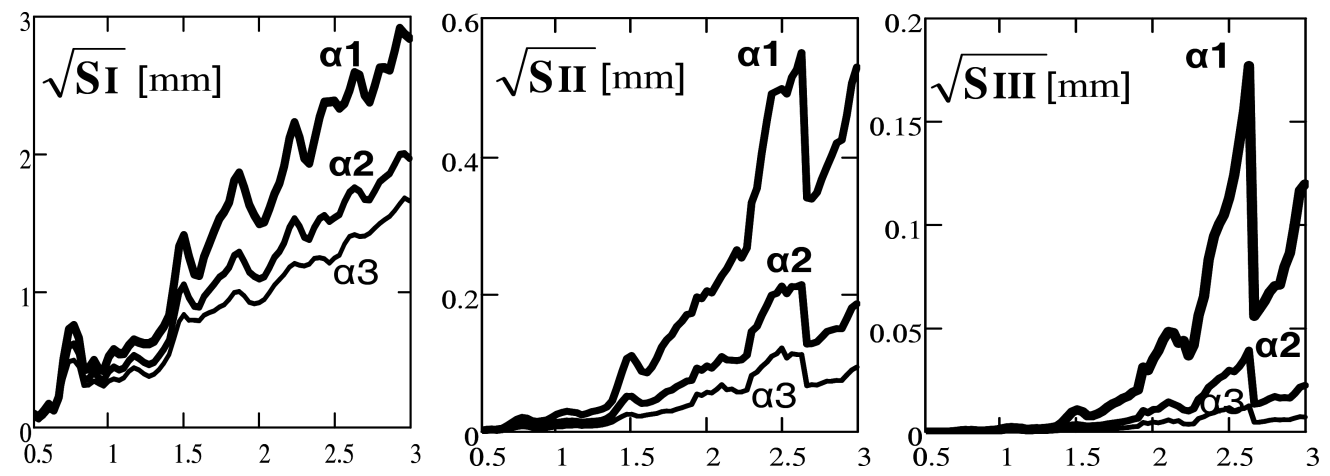

Fig. 4. The backscatter effective cross-section decomposition in respect to the first (SI), second (SII) and third (SIII) scattering order respectively. Plots $\alpha 1, \alpha 2, \alpha 3$ corresponds to the least $(\boldsymbol{\alpha} 1)$, middle $(\boldsymbol{\alpha} 2)$ and largest $(\boldsymbol{\alpha} 3)$ value of the absorption parameter. The frequency in MHz.

a transducer placed close to the scatter. The backscatter (reflection) coefficients can be defined as follows

$$
\kappa_{\mathbf{I}}(n) \equiv \frac{\sqrt{\mathbf{S I}(n)}}{\sqrt{\sigma_{G}}}, \quad \kappa_{\mathbf{I I}}(n) \equiv \frac{\sqrt{\mathbf{S I I}(n)}}{\sqrt{\sigma_{G}}}, \quad \kappa_{\mathbf{I I I}}(n) \equiv \frac{\sqrt{\mathbf{S I I I}(n)}}{\sqrt{\sigma_{G}}},
$$

where $\sigma_{G}$ is the geometrical cross-section (area of the normal projection of the scatter on the surface dividing space into two semi-spaces) for a given configuration. In our case $\sigma_{G}=64 \mathrm{~mm}^{2}$. For the frequency close to $\nu=1.5 \mathrm{MHz}$ the estimated ratio between the values of SI, SII and SIII (see Fig. 4) is 1:0.001: 0.00001 in the case denoted by the $\boldsymbol{\alpha} 1$ and higher in the cases $\boldsymbol{\alpha} 2$ and $\boldsymbol{\alpha} 3$. In Fig. 4 (left) the resonance for $\nu=0.75 \mathrm{MHz}$ is observed. This resonance is created by the $\mathbf{y}$ substructure in which the trabecula length is equal to $2 \mathrm{~mm}$. It corresponds to the resonance frequency. Similar analysis can be performed for other resonances using the higher scattering orders.

In Fig. 5 the contribution of the substructures $\mathbf{h}$ and $\mathbf{y}$ to the backscatter effective cross-sections in the case of $\boldsymbol{\alpha} 1$ for the subsequent orders of scattering is

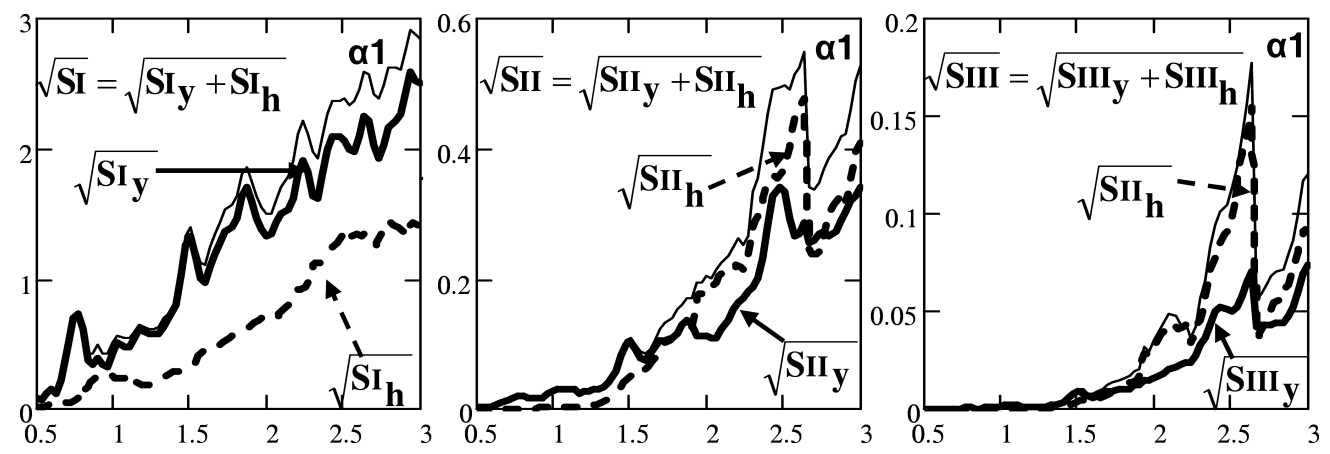

Fig. 5. The contribution of the substructures $\mathbf{h}$ and $\mathbf{y}$ for the subsequent orders of scattering in the case $\boldsymbol{\alpha} 1$ of the absorption parameter as a function of frequency (in $\mathrm{MHz}$ ). 
presented. Let us notice the validity change of the substructures in the transition from the first to the higher orders of scattering. It is visible from Fig. 5 (left and middle) that the transition occurs for the $\nu>1.6 \mathrm{MHz}$.

\section{Conclusions}

In space-frequency range the method of solving the longitudinal wave scattering equations has been developed. It is convergent for high potentials and multi-element structures in numerical applications.

The method is accurate in each order of scattering. It means that the calculated fields in subsequent order do not make corrections in scattering fields of former order. In contrary, the popular Neumann's iteration of the integral equations of scattering produces the asymptotically converged series (if it is converged at all). The developed algorithm enables the analysis of the scattering field characteristics that takes into account the higher orders of scattering. Also it enables to extract the contribution of scattering on the selected group of elements from the total scattering. The examples of this effect for the developed model of the trabecular bone has been presented.

In the frequency range up to $1.5 \mathrm{MHz}$ the influence of the higher scattering orders is minimal and equal to few percent of the first order scattering. In the range above $1.5 \mathrm{MHz}$ one can observe the resonance effects of scattering in higher orders with the magnitude up to twenty percent of first order scattering.

\section{Acknowledgments}

This work is supported in part by Ministry of Science and Higher Education (grants NN 518503339 and NN 518388 234).

\section{References}

1. Bossy E., Padilla F., Peyrin F., Laugier P. (2005), Three-dimensional simulation of ultrasound propagation through trabecular bone structures measured by synchrotron microtomography, Phys. Med. Biol., 50, 5545-5556.

2. Brekhovskikh L.M., Godin O.A. (1990), Acoustics of Layered Media I, ISBN 3-54051038-9, Springer-Verlag Berlin Haidelberg, New York, 1-14.

3. Chaffai S., Roberjot V., Peyrin F., Berger G., Laugier P. (2000), Frequency dependence of ultrasonic backscattering in cancellous bone: Autocorrelation model and experimental results, J. Acoust. Soc. Am., 108, 5, 2403-2411.

4. Hosokawa A. (2008), Development of Numerical Cancellous Bone Model for FiniteDifference Time-Domain Simulations of Ultrasound Propagation, IEEE Trans. Ultrason. Freq. Contr., 55, 6, 1219-1233. 
5. Jackson J.D. (1975), Classical Electrodynamics, Second edition, John Wiley and Sons, Inc., $55-56$.

6. Kaufman J.J., Gangming L., Siffert R.S. (2008), Ultrasound Simulation in Bone, IEEE Trans. Ultrason., Ferr., Freq. Contr., 55, 6, 1205-1217.

7. Laugier P., Talmant M., Pham T. (2008), Que vadis, ultrasonics of bone? Present state and future trends, Archives of Acoustics, 33, 4, 553-564.

8. Litniewski J., Nowicki A., Lewin P.A. (2009), Semi-empirical bone model for determination of trabecular structure properties from backscattered ultrasound, Ultrasonics, 49, $505-513$.

9. Litniewski J. (2010), Statistics of Envelope of High-Frequency Ultrasonic Backscatter From Trabecular Bone: Simulation Study, Archives of Acoustics, 35, 3, 349-360.

10. WEAR K. (1999), Frequency dependence of ultrasonic backscatter from human trabecular bone: theory and experiments, J. Acoust. Soc. Am., 106, 6, 3659-3664.

11. WeAR K. (2008), Ultrasonic Scattering from Cancellous Bone: A review, IEEE Trans. Ultrason. Freq. Contr., 55, 7, 1432-1441.

12. WóJCIK J. (1998), Conservation of energy and absorption in acoustic fields for linear and nonlinear propagation, J. Acoust. Soc. Am., 104, 5, 2654-2663. 